\title{
An old man with swollen right leg
}

\author{
Chung-Ju Yu ${ }^{1}$, Ching-Yi Shen ${ }^{1}$ and Yu-Jang Su, ${ }^{1,2,3 *}$ \\ ${ }^{1}$ Department of Emergency Medicine, Mackay Memorial Hospital, Taipei, Taiwan \\ ${ }^{2}$ Department of Medicine, Mackay Medical College, New Taipei City, Taiwan \\ ${ }^{3}$ Department of Oral Hygiene, College of Oral Medicine, Taipei Medical University, Taipei, Taiwan
}

\begin{abstract}
A 72-year-old man with a history of hypertension presented to the emergency department with increasing swelling and redness of right lower limb for one week. He had a gradually growing mass over right inguinal area for six months. We arranged computed tomography (CT) scans and revealed diffuse enlarged lymph nodes in para-aortic region, right iliac chain (especially right external iliac chain) and right inguinal region, accompanied with right leg swelling and subcutaneous stranding. The Ga-67 citrate whole body scan showed lymphoma involving the right iliac chain and right inguinal regions. Peripheral venous ultrasound revealed external compression over the proximal iliac veins without venous thrombosis. The diagnosis of a diffuse large B cell lymphoma (DLBCL) with lymphedema was confirmed by bone marrow biopsy. He tolerated the chemotherapy well and discharged in good condition one week later.
\end{abstract}

\section{Introduction}

Red swollen leg is commonly seen in emergency department (ED). The common diagnoses including lymphedema, deep venous thrombosis (DVT), and cellulitis. The common symptoms include fever, redness, swollen or leg pain. Here we report a case of right reddish and swollen leg in a 72-year-old man who presented with a six-month history of gradually enlarged inguinal mass. The symptoms were easily considered to be DVT, or cellulitis initially.

\section{Case report}

A 72-year-old man had a history of hypertension presented to our emergency department (ED) with increasing swollen and redness of right lower limb for one week (Figure 1A, white arrow key). He also had a gradually growing mass over right inguinal area for six months. He ever took some herbal medicine, but the inguinal mass grew bigger day by day. He denied fever, skin itching, sweating, body weight loss (BWL) nor trauma history. On physical examination, his body temperature was 37.1 degrees Celsius; blood pressure was 146/84 mm Hg; heart rate was 81 beats per minute and respiratory rate was 20 breaths per minute. His right lower limb was reddish, swollen and huge inguinal lump was palpated (Figure 1B, black arrow key). Homans' sign was negative. Laboratory studies revealed hemoglobin of $15.1 \mathrm{~g} / \mathrm{dl}$, hematocrit of $37.9 \%$ and WBC 5100 /micro-L (Segment: $56.5 \%$, Eosinophil: 9.0 \%, Basophil: $0.7 \%$, Monocyte: $12.3 \%$, and Lymphocyte: $21.5 \%$ ). The computed tomography (CT) scans of abdomen revealed diffuse enlarged lymph nodes in para-aortic region, right iliac chain (especially right external iliac chain) and right inguinal region (Figure 1C, white arrow key). After admission, the bone marrow biopsy was arranged and showed hypercellular marrow with maturing trilineage hematopoiesis low-grade B cell lymphoma involvement (Bc1-6+/MUM-/Bcl-2+). The Ga-67 citrate whole body scan showed lymphoma involving the right iliac chain and right inguinal regions. Peripheral arterial and venous ultrasound revealed external compression over the proximal iliac veins without venous thrombosis and no significant arterial stenosis. The definite diagnosis was diffuse large B cell lymphoma (DLBCL) stage IIA with lymphedema. RCHOP (rituximab, cyclophosphamide, hydroxydaunomycin, vincristine and prednisolone) chemotherapy

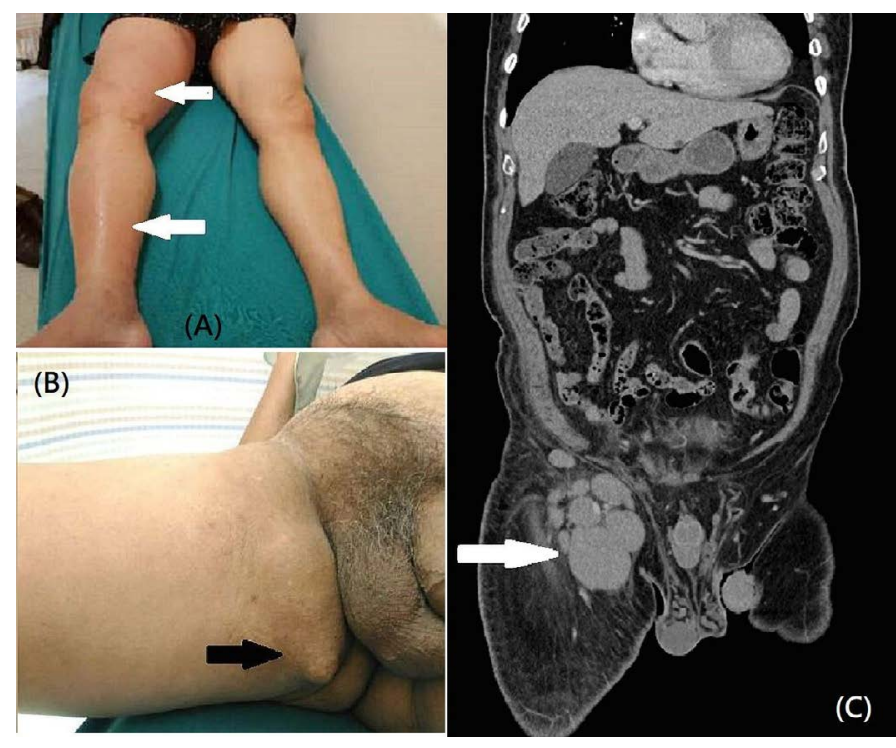

Figure 1. (A) Increasing swollen and redness of right lower limb for one week (white arrow key); (B) Right lower limb was reddish, swollen and huge inguinal lump was palpated (black arrow key); (C) diffuse enlarged lymph nodes in para-aortic region, right iliac chain (especially right external iliac chain) and right inguinal region (white arrow key)

was administrated to treat his $\mathrm{B}$ cell lymphoma. He tolerated the chemotherapy well and was discharged one week later. He had less swollen right leg and regularly follow-up at the outpatient clinic

${ }^{\star}$ Correspondence to: Yu-Jang Su, MD, Department of Emergency Medicine, Mackay Memorial Hospital, Taipei, Taiwan and Department of Oral Hygiene, College of Oral Medicine, Taipei Medical University, Taipei, Taiwan, E-mail: yjsu.5885@mmh.org.tw

Key words: large B cell lymphoma, inguinal lump, chemotherapy

Received: November 19, 2018; Accepted: November 28, 2018; Published: November 30, 2018 
Table 1. Differential diagnosis of cellulitis, erysipelas, lymphedema, deep venous thrombosis, and diffuse large b cell lymphoma

\begin{tabular}{|c|c|c|c|}
\hline Disease & Symptom & Physical examination & Involvement \\
\hline Cellulitis & Fever, red, swollen, hot and painful & Red, tenderness & Topical, blood stream, lymphatic system \\
\hline Erysipelas & $\begin{array}{l}\text { Large, raised red patches on the skin. } \\
\text { Sometimes blisters, fevers, and chills. }\end{array}$ & Red, well-defined margin redness. & $\begin{array}{l}\text { Bacterial infection in the upper layer of } \\
\text { the skin, usually caused by the Group A } \\
\text { Streptococcus spp. Most frequently occurs on } \\
\text { the face and legs. }\end{array}$ \\
\hline Lymphedema & $\begin{array}{l}\text { Fever, reddish, swollen, hot, feeling chills } \\
\text { and painful. }\end{array}$ & $\begin{array}{l}\text { Reddish, swollen, and tenderness. No unique } \\
\text { signs. }\end{array}$ & $\begin{array}{l}\text { Topical limb involved, primary or secondary. } \\
\text { Secondary is more commonly seen by cancer, } \\
\text { surgery, trauma or infection. }\end{array}$ \\
\hline Deep venous thrombosis & $\begin{array}{l}\text { Lower extremity swelling, lower extremity } \\
\text { pain and calf tenderness. }\end{array}$ & Homans' sign may be detectable positively. & $\begin{array}{l}\text { Blood clots in the deep veins, (such as the } \\
\text { femoral vein, popliteal vein, or calf vein) }\end{array}$ \\
\hline Diffuse large B-cell lymphoma & $\begin{array}{l}\text { B symptoms (fever, night sweats, and weight } \\
\text { loss). leg swelling, or edema caused by } \\
\text { extensive pelvic lymphadenopathy (LAP), } \\
\text { fatigue, anorexia, or chest discomfort caused } \\
\text { by mediastinal LAP }\end{array}$ & Rapid growth rate resulting in lumps. & $\begin{array}{l}\text { The clinical manifestations depend on the site } \\
\text { of disease involvement }\end{array}$ \\
\hline
\end{tabular}

\section{Discussion}

Leg swelling is commonly seen in daily practice in the emergency department (ED). The common diagnoses of leg swelling including cellulitis, erysipelas, lymphedema, and deep venous thrombosis. Emergency physicians make diagnosis differentially from variate presentations and clinical courses of above-mentioned diseases. Here we presented a case of diffuse large B cell lymphoma (DLBCL) mimicking cellulitis initially. Red swollen leg is a condition commonly seen in patients in ED. The differential diagnosis includes cellulitis, erysipelas, deep vein thrombosis (DVT), or lymphedema. Cellulitis involves the dermis and subcutaneous tissues of the skin due to the bacterial infection [1]. The symptoms of cellulitis include hot, painful, red, tender and swollen area of skin. Erysipelas only involves the upper dermis, characteristically extending into the superficial cutaneous lymphatics. Erysipelas has a well-defined border, differing from other skin infections $[1,2]$. The pathophysiology of DVT is the formation of blood clots in the deep veins, commonly affecting the deep leg veins (such as the femoral vein, popliteal vein, or calf vein) [3]. DVT may present with lower extremity swelling, lower extremity pain and calf tenderness. Homans' sign may be detectable positively in DVT. Most of the symptoms and signs are not specific. Compression venous ultrasound has proven to be a highly sensitivity and specificity for the diagnosis of lower extremity DVT [4]. Lymphedema is clinically characterized with chronic swelling, skin changes, localized pain and secondary infections. Lymphedema is caused by impaired lymphatic drainage. The impairment of the lymphatic system causes tissue swelling due to excessive retention of fluid in the interstitial compartment [5]. Lymphedema can be either primary or secondary. Secondary lymphedema is more common than primary lymphedema. The causes of secondary lymphedema include cancer, surgery, trauma or infection [5]. Because no unique signs or symptoms for lymphedema, the diagnosis is usually made clinically by thorough physical examination and evaluation. Lymphomas are the most common type of blood cancers. There are two types of lymphoma: non-Hodgkin's and Hodgkin's. Diffuse large B-cell lymphoma (DLBCL) is the most commonly seen non-Hodgkin's lymphoma. DLBCL is the most aggressive or fast-growing form [6]. The clinical manifestations depend on the site of disease involvement. The growth rate of the DLBCL is rapid and results in lumps. The B symptoms of DLBCL include fever, night sweats, and weight loss [6]. Other symptoms can include the following: leg swelling, or edema caused by extensive pelvic lymphadenopathy, fatigue, anorexia, or chest discomfort caused by mediastinal lymphadenopathy (LAP) [7]. The surviving rate of DLBCL depends on many factors. The following factor reduce the chances of survival: age older than 60 , lactate dehydrogenase (LDH) level higher than normal, poor general health status, (Eastern cooperative oncology group (ECOG) performance status score of 2 or greater), stage III or IV disease and more than one involved extranodal disease site [7]. Pain in limb with reddish, swollen appearance is commonly seen in ED, and emergency physicians need to make differential diagnosis carefully. The detailed distinguish list is in (Table 1).

\section{Conclusion}

Red swollen legs are commonly seen in ED and commonly diagnosed as cellulitis, erysipelas, lymphedema, and DVT. Large B cell lymphoma of leg is relative uncommon, but physicians need to keep in mind to list in differential diagnoses. To prudently see patients including inguinal area is important to perform further images studies, and then made the accurate diagnosis.

\section{Conflicts of interest}

There are no conflicts of interest.

\section{References}

1. Bailey E, Kroshinsky D (2011) Cellulitis: diagnosis and management. Dermatol Ther 24: 229-239. [Crossref]

2. Maxwell-Scott H, Kandil H (2015) Diagnosis and management of cellulitis and erysipelas. Br J Hosp Med (Lond) 76: 114-117. [Crossref]

3. Merli GJ (2008) Pathophysiology of venous thrombosis and the diagnosis of deep vein thrombosis-pulmonary embolism in the elderly. Cardiol Clin 26: 203-219. [Crossref]

4. Bernardi E, Camporese G (2017) Diagnosis of deep-vein thrombosis. Thromb Res 3848: 30522-30524.

5. Grada AA, Phillips TJ (2017) Lymphedema: pathophysiology and clinical manifestations. J Am Acad Dermatol 77: 1009-1020. [Crossref]

6. Li S, Young KH, Medeiros LJ (2018) Diffuse large B-cell lymphoma. Pathology 50: 74-87.

7. Ziepert M, Hasenclever D, Kuhnt E (2010) Standard international prognostic index remains a valid predictor of outcome for patients with aggressive CD20 + B-cell lymphoma in the rituximab era. J Clin Oncol 28: 2373-2380. [Crossref]

Copyright: (C2018 Yu C. This is an open-access article distributed under the terms of the Creative Commons Attribution License, which permits unrestricted use, distribution, and reproduction in any medium, provided the original author and source are credited. 